\title{
Adaptive Global Sliding Mode Control for MEMS Gyroscope Using RBF Neural Network
}

\author{
Yundi Chu and Juntao Fei \\ College of IOT Engineering, Hohai University, Changzhou 213022, China \\ Correspondence should be addressed to Juntao Fei; jtfei@yahoo.com
}

Received 8 September 2014; Accepted 3 March 2015

Academic Editor: Xinkai Chen

Copyright ( $) 2015$ Y. Chu and J. Fei. This is an open access article distributed under the Creative Commons Attribution License, which permits unrestricted use, distribution, and reproduction in any medium, provided the original work is properly cited.

\begin{abstract}
An adaptive global sliding mode control (AGSMC) using RBF neural network (RBFNN) is proposed for the system identification and tracking control of micro-electro-mechanical system (MEMS) gyroscope. Firstly, a new kind of adaptive identification method based on the global sliding mode controller is designed to update and estimate angular velocity and other system parameters of MEMS gyroscope online. Moreover, the output of adaptive neural network control is used to adjust the switch gain of sliding mode control dynamically to approach the upper bound of unknown disturbances. In this way, the switch item of sliding mode control can be converted to the output of continuous neural network which can weaken the chattering in the sliding mode control in contrast to the conventional fixed gain sliding mode control. Simulation results show that the designed control system can get satisfactory tracking performance and effective estimation of unknown parameters of MEMS gyroscope.
\end{abstract}

\section{Introduction}

MEMS gyroscope is a sensor which has many advantages such as small size, low price, and low energy consumption. So it has a promising application potential in many fields, widely used not only in aviation, aerospace, and marine but also in weapons, cars, robots, and so on owing to its remarkable features. However, MEMS gyroscope also has some disadvantages on the other hand. As a matter of fact, small manufacture error always exists because of the limitations of the design principle and the machining accuracy. In the recent years, many advanced control approaches have been proposed to resolve a series of shortcomings and make MEMS gyroscope have a better performance. For instance, Batur et al. [1] introduced a sliding mode control with force balanced and adaptive method for MEMS gyroscope. Leland [2] proposed a Lyapunov based adaptive control for a MEMS gyroscope. Fei et al. [3] presented a scheme of using adaptive sliding mode robust tracking control for triaxial angular velocity sensors. Sung et al. [4] developed the control of MEMS gyroscope through phase-domain analysis and design. John and Vinay [5] proposed a controlled single mass triaxial angular rate sensor.
Global sliding mode control has been vastly applied into practice since it can ensure the robustness and speed ability of the system and eliminate the reaching time of sliding mode surface. Liu et al. [6] realized application of global sliding mode control in chaotic systems. Efimov and Fridman [7] presented a global sliding mode observer for locally Lipschitz systems with adjusted gains. Chu and Fei [8] proposed a global sliding mode control of MEMS gyroscope using neural network to improve the robustness of the system and reduce chattering.

Adaptive sliding mode control is an important way since it has numerous highlighted features like estimation of the unknown system parameters. On account of uncertainties and disturbances in reality, some intelligent control methods such as fuzzy control and neural network have been applied to various kinds of nonlinear systems more and more. Rastovic [9] presented some compensators for contractive systems. Zhang [10] derived a discrete adaptive sliding mode controller for a class of nonlinear systems by wavelet network. Lin and Li [11] designed an adaptive dynamic fuzzy sliding mode CMAC using asymmetric Gaussian function for voice coil motor. Fei and Ding [12] developed an adaptive sliding mode control for dynamic system using RBF neural network. 
Rastovic [13] proposed an adaptive recurrent neural networks synchronization of $\mathrm{H}$-mode and edge localized modes that is important for obtaining a long-pulse tokamak without disruption regime. Fei and Zhou [14] introduced an adaptive fuzzy compensator for MEMS gyroscope. Lin and Chen [15] employed adaptive hybrid type 2 intelligent sliding mode control for uncertain nonlinear multivariable dynamical systems. Oong and Isa [16] proposed an adaptive neural network which is evolutionary for pattern classification. Zhang and $\mathrm{Ge}$ [17] used an adaptive tracking control of MIMO nonlinear systems based on neural network with unknown control directions and dead zones. Zhao et al. [18] presented a method of consensus control using neural networks for multiple robotic manipulators. An adaptive neural network control is designed for small rotary-wing unmanned aircraft by Lei and $\mathrm{Lu}$ [19]. Zou et al. [20] used terminal sliding mode and Chebyshev neural network finite-time attitude tracking control for spacecraft. Lou and Cui [21] utilized a robust adaptive synchronization control based on chaotic neural networks by slide technique. Chen [22] derived a robust adaptive control with structure neural-fuzzy network for robot manipulators.

We develop a scheme of adaptive global sliding mode control using neural network in this paper. For one thing, global sliding mode control guarantees the stability and robustness of the system and has a faster control effect compared to sliding mode control. Adaptive global sliding mode control can adjust and estimate the angular velocity and other parameters online. For another thing, neural network is good at estimating the upper bound of uncertainties and disturbances and decreasing chattering for MEMS gyroscope. The combination of adaptive global sliding mode and RBF neural networks for gyroscope plays their advantages separately to improve the performance of the system. The proposed strategy has the following advantages.

(1) The transient characteristics and robustness of the sliding mode control system can be improved by selecting suitable sliding coefficient. And it also makes the closedloop control system have a global robustness with resolving the contradiction between transient performance and robustness. An RBF neural network is used to adaptively approximate the unknown upper bound of disturbance to eliminate the chattering effectively.

(2) The global sliding mode control, adaptive control, and the nonlinear approximation of neural network control are successfully integrated to control the MEMS gyroscope. Compared with global sliding mode control, adaptive global sliding mode control based on neural network can not only make the system reach the sliding surface more quickly but also eliminate the chattering that exists during the sliding control.

This paper can be arranged as follows. We establish mathematical model of gyroscope in Section 2. In Section 3, an adaptive global sliding mode control approach is presented to estimate the unknown parameters of the system. Furthermore, adaptive global sliding mode control using neural networks is utilized to approach the upper bound of system uncertainties and disturbances in Section 4. At the same time the chattering of the control input can also be

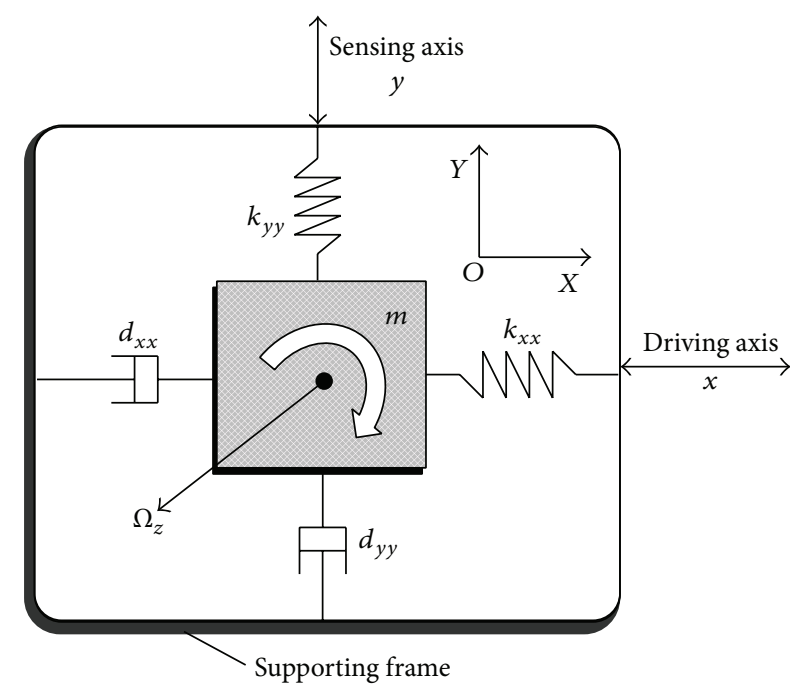

FIGURE 1: Structure of $z$-axis MEMS gyroscope.

reduced. Section 5 shows the satisfactory simulation results. Conclusions are given in the last section.

\section{Mathematical Model of MEMS Gyroscope}

This part introduces the mathematical model of microvibration gyroscope. The simplified model of MEMS gyroscope is shown in Figure 1. It is a mass-spring-damper system. Gyroscope can be simplified to a damped oscillation system composed of a mass and spring. We decompose the movement into two directions and ignore the linear acceleration of the framework of gyroscope. Then we can get the vibratory equation as follows:

$$
\begin{aligned}
& m \ddot{x}+d_{x x} \dot{x}+d_{x y} \dot{y}+k_{x x} x+k_{x y} y=u_{x}+d_{x}+2 m \Omega_{z} \dot{y} \\
& m \ddot{y}+d_{x y} \dot{x}+d_{y y} \dot{y}+k_{x y} x+k_{y y} y=u_{y}+d_{y}-2 m \Omega_{z} \dot{x}
\end{aligned}
$$

where the mass of the mass block is $m$; the damping constants are $d_{x x}, d_{y y}$ and spring constants are $k_{x x}, k_{y y}$; coupling elastic coefficient and damping coefficient caused by the manufacturing error are $d_{x y}, k_{x y}$; control inputs of the axles are $u_{x}, u_{y}$; the mechanical noise and disturbances in the environment are $d_{x}, d_{y} . \Omega_{z}$ is angular velocity in the $z$ direction.

Nondimensional trajectory $q^{*}$ can be replaced by $q^{*}=$ $q / q_{0}$ and nondimensional time $t^{*}$ can also be replaced by $t^{*}=\omega_{0} t$. (1) can be divided by the square of the resonant frequency, the mass, and the reference length at the same time on both sides. We get dimensionless vector form of dynamic equation of MEMS gyroscope as follows:

$$
\ddot{q}^{*}+D^{*} \dot{q}^{*}+K^{*} q^{*}=u^{*}-2 \Omega^{*} \dot{q}^{*}
$$


where

$$
\begin{gathered}
D^{*}=\frac{D}{m \omega_{0}}, \quad D=\left[\begin{array}{ll}
d_{x x} & d_{x y} \\
d_{x y} & d_{y y}
\end{array}\right], \\
K^{*}=\left[\begin{array}{ll}
\omega_{x}^{2} & \omega_{x y} \\
\omega_{x y} & \omega_{y}^{2}
\end{array}\right], \quad u^{*}=\frac{u}{m \omega_{0}^{2} q_{0}}, \\
\omega_{x}=\sqrt{\frac{k_{x x}}{m \omega_{0}^{2}}}, \quad \omega_{y}=\sqrt{\frac{k_{y y}}{m \omega_{0}^{2}}} \\
\omega_{x y}=\frac{k_{x y}}{m \omega_{0}^{2}}, \quad u=\left[\begin{array}{l}
u_{x} \\
u_{y}
\end{array}\right], \\
\Omega^{*}=\frac{\Omega}{\omega_{0}}, \quad \Omega=\left[\begin{array}{cc}
0 & -\Omega_{z} \\
\Omega_{z} & 0
\end{array}\right] .
\end{gathered}
$$

We replace $q^{*}$ with $q, t^{*}$ with $t, D^{*}$ with $D, K^{*}$ with $K, u^{*}$ with $u, \Omega^{*}$ with $\Omega$, so the final form of the dimensionless equation may be described as

$$
\ddot{q}+D \dot{q}+K q=u-2 \Omega \dot{q},
$$

where $q$ is trajectory of MEMS gyroscope and $u$ is control input.

\section{Adaptive Global Sliding Mode Control}

In this section, when the external disturbances and parameter uncertainties are taken into account, we proposed an adaptive global sliding mode control method to estimate the angular velocity and other parameters of MEMS gyroscopes.

Rewriting (4) in state-space equation, we obtain

$$
\dot{X}=A X+B u,
$$

where

$$
\begin{aligned}
& X=\left[\begin{array}{c}
x \\
\dot{x} \\
y \\
\dot{y}
\end{array}\right] \\
& A=\left[\begin{array}{cccc}
0 & 1 & 0 & 0 \\
-\omega_{x}^{2} & -d_{x x} & -\omega_{x y} & -\left(d_{x y}-2 \Omega_{z}\right) \\
0 & 0 & 0 & 1 \\
-\omega_{x y} & -\left(d_{x y}+2 \Omega_{z}\right) & -\omega_{y}^{2} & -d_{y y}
\end{array}\right] \text {, } \\
& B=\left[\begin{array}{ll}
0 & 0 \\
1 & 0 \\
0 & 0 \\
0 & 1
\end{array}\right], \quad u=\left[\begin{array}{l}
u_{x} \\
u_{y}
\end{array}\right] .
\end{aligned}
$$

Because the ideal dynamic characteristic of MEMS gyroscope is a kind of no energy loss and there is no unstable sinusoidal oscillation which is dynamic coupling between the two axes, it can be described as $x_{m}=A_{1} \sin \left(\omega_{1} t\right), y_{m}=A_{2} \sin \left(\omega_{2} t\right)$.

We define the reference model a: $\ddot{q}_{m}+K_{m} q_{m}=0$, where $K_{m}=\operatorname{diag}\left\{\omega_{1}^{2} \omega_{2}^{2}\right\}$.

The state-space expression is

$$
\dot{X}_{m}=A_{m} X_{m},
$$

where

$$
X=\left[\begin{array}{l}
x_{m} \\
\dot{x}_{m} \\
y_{m} \\
\dot{y}_{m}
\end{array}\right], \quad A=\left[\begin{array}{cccc}
0 & 1 & 0 & 0 \\
-\omega_{1}^{2} & 0 & 0 & 0 \\
0 & 0 & 0 & 1 \\
0 & 0 & -\omega_{2}^{2} & 0
\end{array}\right], \quad \omega_{1} \neq \omega_{2} .
$$

Considering the system with parameter uncertainties and external disturbances, we convert (5) into

$$
\dot{X}(t)=A X(t)+B u+f_{m}(t),
$$

where $f_{m}(t)$ is lumped parameter uncertainties and external disturbances.

We make the following assumptions.

Assumption 1. There exists known positive constant $\partial_{m}$ to make the lumped parameter uncertainties and external disturbances meet the upper bound condition:

$$
\left\|f_{m}(t)\right\| \leq \partial_{m} .
$$

Assumption 2. There always exists $K^{*}$ which is a constant matrix to satisfy the equation $A+B K^{* T}=A_{m}$.

Then we can get

$$
K^{* T}=\left(B^{T} B\right)^{-1} B^{T}\left(A_{m}-A\right) .
$$

Define the tracking error:

$$
e(t)=X(t)-X_{m}(t) .
$$

And its derivative is

$$
\dot{e}=A_{m} e+\left(A-A_{m}\right) X+B u+f_{m} .
$$

Define global sliding surface as

$$
s=\dot{e}+C e-f(t),
$$

where $C$ is sliding coefficient and $f(t)$ is a function that is specially designed for reaching the global sliding surface, satisfying the following three conditions:

(1) $f(0)=\dot{e}_{0}+C e_{0}$;

(2) if $t \rightarrow \infty, f(t) \rightarrow 0$;

(3) $f(t)$ has a first derivative.

where $e_{0}$ is an initial value of the tracking error.

Therefore we can design $f(t)$ as $f(t)=f(0) e^{-k t}$, where $k$ is a constant. 


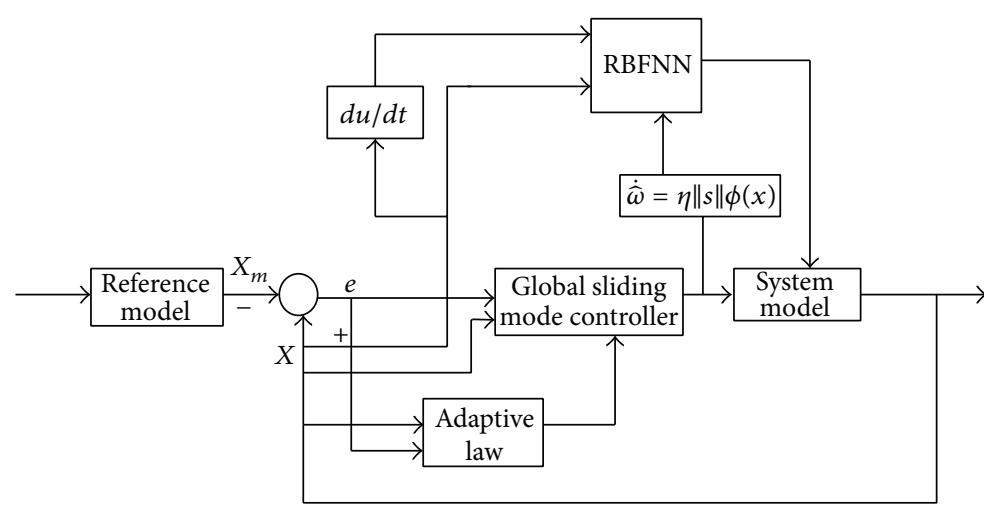

FIGURE 2: Block diagram of AGSMC using RBFNN for the MEMS gyroscope. $f_{e}(t)$.

We define $\dot{e}-f(t)=f_{e}(t)$; then (14) becomes $s=C e+$

The derivative of $s$ is

$$
\begin{aligned}
\dot{\boldsymbol{s}} & =C \dot{e}+\dot{f}_{e}(t) \\
& =C\left[A_{m} e+\left(A-A_{m}\right) X+B u+f_{m}\right]+\dot{f}_{e}(t) \\
& =C\left[A_{m} e-B K^{* T} X+B u+f_{m}\right]+\dot{f}_{e}(t) \\
& =C A_{m} e-C B K^{* T} X+C B u+C f_{m}+\dot{f}_{e}(t) .
\end{aligned}
$$

Set $\dot{s}=0$ to solve the equivalent control as

$$
u_{\mathrm{eq}}=K^{* T} X-(C B)^{-1} C A_{m} e-(C B)^{-1} C f_{m}-(C B)^{-1} \dot{f}_{e}(t) \text {. }
$$

On account of unknown parameters of $A$, so $K^{*}$ is unknown. Therefore, we can replace $K^{*}$ by its estimate value $K$. The system may be adjusted adaptively to identify the unknown parameters.

Design the following adaptive control law:

$$
u=K^{T} X-(C B)^{-1} C A_{m} e-\rho(C B)^{-1} \frac{s}{\|s\|}-(C B)^{-1} \dot{f}_{e}(t)
$$

where $K$ is the estimate value of $K^{*}$ and switching gain $\rho$ is used to compensate for uncertain items $f_{m}$ to ensure that the sliding mode existence conditions are met.

Hence, (15) can be transformed into

$$
\dot{s}=C B \widetilde{K}^{T} X+C f_{m}-\rho \frac{s}{\|s\|},
$$

where $\widetilde{K}=K-K^{*}$ is the estimation error.

Define a Lyapunov function candidate:

$$
V_{1}=\frac{1}{2} s^{T} s+\frac{1}{2} \operatorname{tr}\left[\widetilde{K} M^{-1} \widetilde{K}^{T}\right],
$$

where $M=M^{T}>0, M$ is a positive definite matrix, and $\operatorname{tr}[M]$ is the trace of $M$.
Then the derivative of (19) becomes

$$
\begin{aligned}
\dot{V}_{1} & =s^{T} \dot{s}+\operatorname{tr}\left[\widetilde{K} M^{-1} \dot{\widetilde{K}}^{T}\right] \\
& =s^{T}\left[C B \widetilde{K}^{T} X+C f_{m}-\rho \frac{s}{\|s\|}\right]+\operatorname{tr}\left[\widetilde{K} M^{-1} \dot{\widetilde{K}}^{T}\right] \\
& =s^{T} C B \widetilde{K}^{T} X+s^{T} C f_{m}-s^{T} \rho \frac{s}{\|s\|}+\operatorname{tr}\left[\widetilde{K} M^{-1} \dot{\widetilde{K}}^{T}\right] \\
& =\operatorname{tr}\left[\widetilde{K} B^{T} C^{T} s X^{T}\right]+\operatorname{tr}\left[\widetilde{K} M^{-1} \dot{\widetilde{K}}^{T}\right]+s^{T} C f_{m}-\rho\|s\| .
\end{aligned}
$$

The sum of the first two items in (20) should be equal to zero to meet $\dot{V}_{1} \leq 0$. As a result, we choose the following adaptive law:

$$
\dot{\widetilde{K}}^{T}(t)=\dot{K}^{T}(t)=-M B^{T} C^{T} s X^{T} .
$$

Substituting $\dot{\widetilde{K}}^{T}(t)$ in (21) into (20), we obtain

$$
\begin{aligned}
\dot{V}_{1} & =s^{T} C f_{m}-\rho\|s\| \leq\|s\|\|C\|\left\|f_{m}\right\|-\rho\|s\| \\
& \leq\|s\|\|C\| \partial_{m}-\rho\|s\|=-\|s\|\left(\rho-\|C\| \partial_{m}\right) \leq 0
\end{aligned}
$$

where $\rho \geq\|C\| \partial_{m}+\eta_{1}$ and $\eta_{1}$ is a positive constant.

Because $f_{m}$ is unknown, $\rho$ is difficult to be confirmed. It may not be enough to eliminate the influence of the uncertainties and cause instability of the system if $\rho$ is too small. It may also cause large chattering if $\rho$ is too large. In order to solve such problem, adaptive global sliding mode control using neural network is proposed further based on parameter identification. Switching gain in the switch item of sliding mode control is estimated continuously and effectively to reduce the chattering.

\section{Adaptive Global Sliding Mode Control Using Neural Network}

We adopt adaptive neural system to approach the switching gain as shown in Figure 2. We suppose that the upper bound 


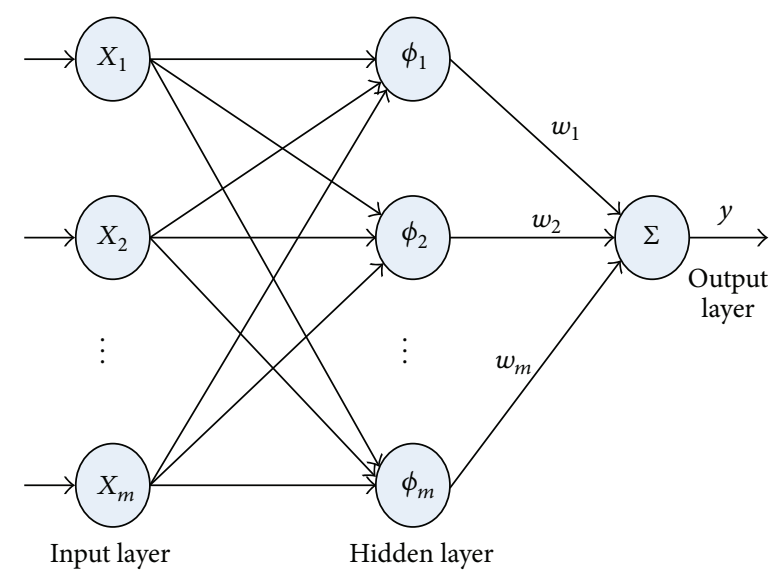

FIGURE 3: The structure of RBF neural network.

of lumped system uncertainties and external disturbances is $\bar{\rho}$. We should make use of RBF neural network to study the upper bound $\bar{\rho}$ adaptively owing to the unknown $\bar{\rho}$. The estimate value of $\bar{\rho}$ is $\hat{\bar{\rho}}(x, \omega)=\widehat{\omega}^{T} \phi(x)$. The structure of RBF neural network is shown in Figure 3, where $X$ and $y$ are the input and output of neural network, respectively; the estimated weights vector is $\widehat{\omega}$; the weights vectors are $\omega=\left[\omega_{1}, \omega_{2}, \ldots, \omega_{n}\right]^{T} . \phi(x)=\left[\phi_{1}(x), \phi_{2}(x), \ldots, \phi_{n}(x)\right]^{T}$ are Gaussian functions.

We make the following assumptions.

Assumption 3. The weights of neural network satisfy

$$
\omega^{* T} \phi-\bar{\rho}=\xi, \quad|\xi|<\xi_{1} .
$$

Assumption 4. The upper bound $\bar{\rho}$ meets

$$
\bar{\rho}-\|C\|\left\|f_{m}\right\|>\xi_{0}>\xi_{1} .
$$

Design the adaptive controller with RBF neural network as

$$
u=K^{T} X-(C B)^{-1} C A_{m} e-\hat{\bar{\rho}}(C B)^{-1} \frac{s}{\|s\|}-(C B)^{-1} \dot{f}_{e}(t) .
$$

Then the dynamics of sliding surface become

$$
\dot{s}=C B \widetilde{K}^{T} X+C f_{m}-\overline{\bar{\rho}} \frac{s}{\|s\|} .
$$

Define a Lyapunov function candidate:

$$
V=\frac{1}{2} s^{T} s+\frac{1}{2} \operatorname{tr}\left[\widetilde{K} M^{-1} \widetilde{K}^{T}\right]+\frac{1}{2 \eta} \widetilde{\omega}^{T} \widetilde{\omega},
$$

where $\widetilde{\omega}=\omega^{*}-\widehat{\omega}, \eta=\xi_{0}-\xi_{1}>0, M=M^{T}>0, M$ is a positive definite matrix, and $\operatorname{tr}[M]$ is the trace of $M$.
The derivative of (27) is

$$
\begin{aligned}
\dot{V}= & s^{T} \dot{\boldsymbol{s}}+\operatorname{tr}\left[\widetilde{K} M^{-1} \dot{\tilde{K}}^{T}\right]+\frac{1}{\eta} \widetilde{\omega}^{T} \dot{\tilde{\omega}} \\
= & s^{T}\left[C B \widetilde{K}^{T} X+C f_{m}-\hat{\bar{\rho}} \frac{s}{\|s\|}\right]+\operatorname{tr}\left[\widetilde{K} M^{-1} \dot{\tilde{K}}^{T}\right]+\frac{1}{\eta} \widetilde{\omega}^{T} \dot{\tilde{\omega}} \\
= & \operatorname{tr}\left[\widetilde{K} B^{T} C^{T} s X^{T}\right]+\operatorname{tr}\left[\widetilde{K} M^{-1} \dot{\widetilde{K}}^{T}\right]+s^{T} C f_{m} \\
& -\hat{\bar{\rho}} s^{T} \frac{s}{\|s\|}+\frac{1}{\eta} \widetilde{\omega}^{T} \dot{\tilde{\omega}} .
\end{aligned}
$$

Substituting the parameter adaptive laws (21) into (28) yields

$$
\begin{aligned}
\dot{V} & =s^{T} C f_{m}-\hat{\bar{\rho}}\|s\|+\frac{1}{\eta} \mu \widetilde{\omega}^{T} \dot{\tilde{\omega}} \leq\|s\|\left(\|C\|\left\|f_{m}\right\|-\hat{\bar{\rho}}\right)+\frac{1}{\eta} \widetilde{\omega}^{T} \dot{\tilde{\omega}} \\
& =\|s\|\left(\|C\|\left\|f_{m}\right\|+\bar{\rho}-\bar{\rho}-\overline{\bar{\rho}}\right)+\frac{1}{\eta} \widetilde{\omega}^{T} \dot{\tilde{\omega}} \\
& =-\|s\|\left(\bar{\rho}-\|C\|\left\|f_{m}\right\|\right)+\|s\|(\bar{\rho}-\rho)+\frac{1}{\eta} \widetilde{\omega}^{T} \dot{\tilde{\omega}} \\
& =-\|s\|\left(\bar{\rho}-\|C\|\left\|f_{m}\right\|\right)+\|s\|\left(\omega^{* T} \phi-\xi-\widehat{\omega}^{T} \phi\right)+\frac{1}{\eta} \widetilde{\omega}^{T} \dot{\tilde{\omega}} \\
& =-\|s\|\left(\bar{\rho}-\|C\|\left\|f_{m}\right\|\right)-\|s\| \xi+\left[\|s\| \widetilde{\omega}^{T} \phi+\frac{1}{\eta} \widetilde{\omega}^{T} \dot{\tilde{\omega}}\right] .
\end{aligned}
$$

To make $\dot{V} \leq 0$, we choose the adaptive law as

$$
\dot{\hat{\omega}}=-\dot{\tilde{\omega}}=\eta\|s\| \phi(x) .
$$

Then (29) becomes

$$
\begin{aligned}
\dot{V} & =-\|s\|\left(\bar{\rho}-\|C\|\left\|f_{m}\right\|\right)-\|s\| \xi \\
& \leq-\|s\| \xi_{0}+\|s\| \xi \leq-\|s\| \xi_{0}+\|s\| \xi_{1} \\
& =-\|s\|\left(\xi_{0}-\xi_{1}\right)=-\eta\|s\| \leq 0 .
\end{aligned}
$$

$\dot{V}$ is negative and semidefinite ensuring that $V, s$, and $\widetilde{K}$ are all bounded. $\dot{s}$ is also bounded. Inequality (31) implies that $s$ is integrable as $\int_{0}^{t}\|s\| d t \leq(1 / \eta)[V(0)-V(t)]$; it can be concluded that $\lim _{t \rightarrow \infty} \int_{0}^{t}\|s\| d t$ is bounded. Since $\lim _{t \rightarrow \infty} \int_{0}^{t}\|s\| d t$ is bounded and $\dot{s}$ is also bounded, according to Barbalat lemma, $s(t)$ will asymptotically converge to zero and $\lim _{t \rightarrow \infty} s(t)=0, e(t)$ also converges to zero asymptotically. 

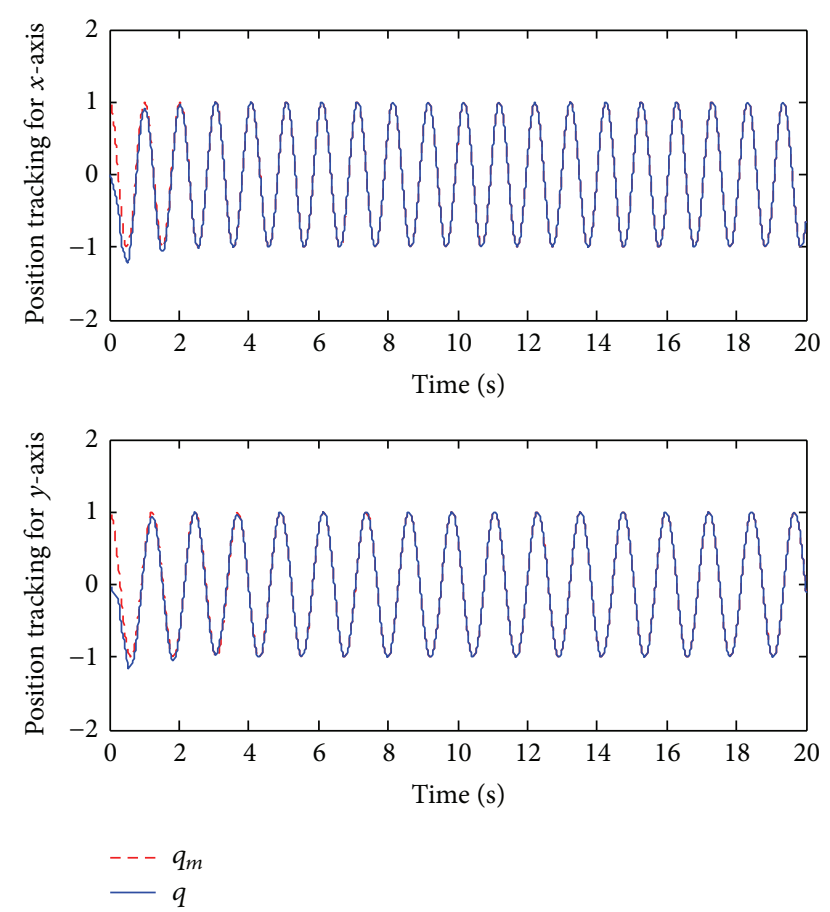

Figure 4: Position tracking using adaptive global sliding neural control.

\section{Simulation Study}

To prove the effectiveness of adaptive global sliding mode control using neural network, simulation study is implemented and demonstrated. The following parameters are selected:

$$
\begin{gathered}
m=1.8 \times 10^{-7} \mathrm{~kg}, \quad k_{x x}=63.955 \mathrm{~N} / \mathrm{m}, \\
k_{y y}=95.92 \mathrm{~N} / \mathrm{m}, \quad k_{x y}=12.779 \mathrm{~N} / \mathrm{m}, \\
d_{x x}=1.8 \times 10^{-6} \mathrm{Ns} / \mathrm{m}, \quad d_{y y}=1.8 \times 10^{-6} \mathrm{Ns} / \mathrm{m}, \\
d_{x y}=3.6 \times 10^{-7} \mathrm{Ns} / \mathrm{m}, \\
q_{0}=1 \mu \mathrm{m}, \quad \omega_{0}=1000 \mathrm{~Hz} .
\end{gathered}
$$

Suppose the angular velocity is $\Omega_{z}=100 \mathrm{rad} / \mathrm{s}$; we get the following parameters after dimensionless procedure:

$$
\begin{gathered}
\omega_{x}^{2}=355.3, \quad \omega_{y}^{2}=532.9, \quad \omega_{x y}=70.99, \\
d_{x x}=0.01, \quad d_{y y}=0.01, \\
d_{x y}=0.002, \quad \Omega_{z}=0.1 .
\end{gathered}
$$

The ideal trajectories of two axes are $q_{d x}=\sin (6.17 t), q_{d y}=$ $\sin (5.11 t)$.

The uncertainty and disturbance can be adopted as $[\operatorname{rand} n(1,1) ; \operatorname{rand} n(1,1)]$.
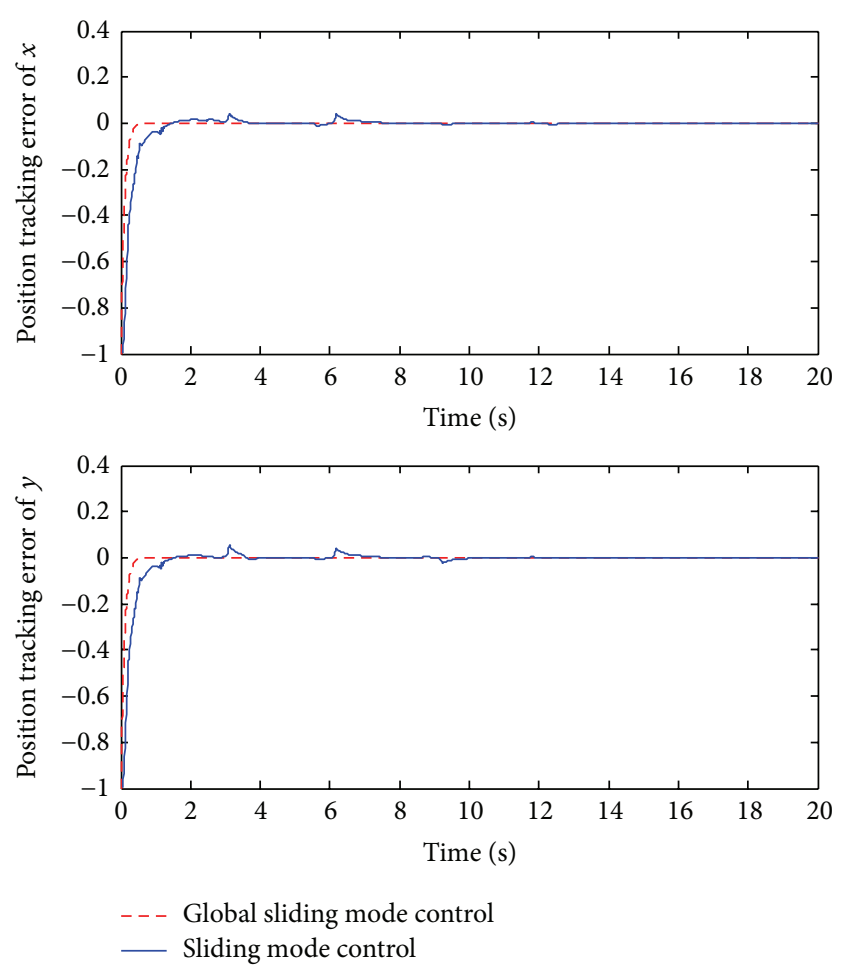

FIGURE 5: Comparison of tracking error between adaptive global sliding mode control using neural network and adaptive sliding mode control using neural network.

Choosing $f(t)=s(0) e^{-50 t}, x(0)=[0,0,0,0]^{T}$, and $K(0)=$ $0.95 K^{*}$, truth values of $K$ are

$$
\begin{aligned}
K^{* T} & =\left[\begin{array}{llll}
k_{11}^{*} & k_{21}^{*} & k_{31}^{*} & k_{41}^{*} \\
k_{12}^{*} & k_{22}^{*} & k_{32}^{*} & k_{42}^{*}
\end{array}\right] \\
& =\left[\begin{array}{llll}
317.2311 & 0.0100 & 70.9900 & -0.1980 \\
70.9900 & 0.2020 & 506.7879 & 0.0100
\end{array}\right] .
\end{aligned}
$$

Sliding mode parameter $C$ is $C=\left[\begin{array}{llll}5 & 1 & 0 & 0 \\ 0 & 0 & 5 & 1\end{array}\right]$, the adaptive gain $M$ is $M=\operatorname{diag}\left[\begin{array}{ll}30 & 30\end{array}\right]$, and the fixed gain $\rho$ in traditional sliding mode control is $\rho=\operatorname{diag}\left[\begin{array}{ll}100 & 100\end{array}\right]$.

The position tracking of two axes using adaptive global sliding neural control is shown in Figure 4. We can find that the real trajectory can track the reference trajectory well in the presence of lumped parameter uncertainties and external disturbances. Moreover, the actual trajectory coincides exactly with the desired one in about 3 minutes, illustrating that the tracking performance is at a preferable state of the AGSMCNN system.

Figure 5 compares tracking errors between adaptive global sliding mode control using neural network and adaptive sliding mode control using neural network. The tracking errors converge to zero more quickly and smoothly using adaptive global sliding mode control, which proves that the former method can achieve better tracking results. From it, we can conclude that global sliding mode control is prior to sliding mode control which should be seen via its good points such as fast speed and strong global robustness. 

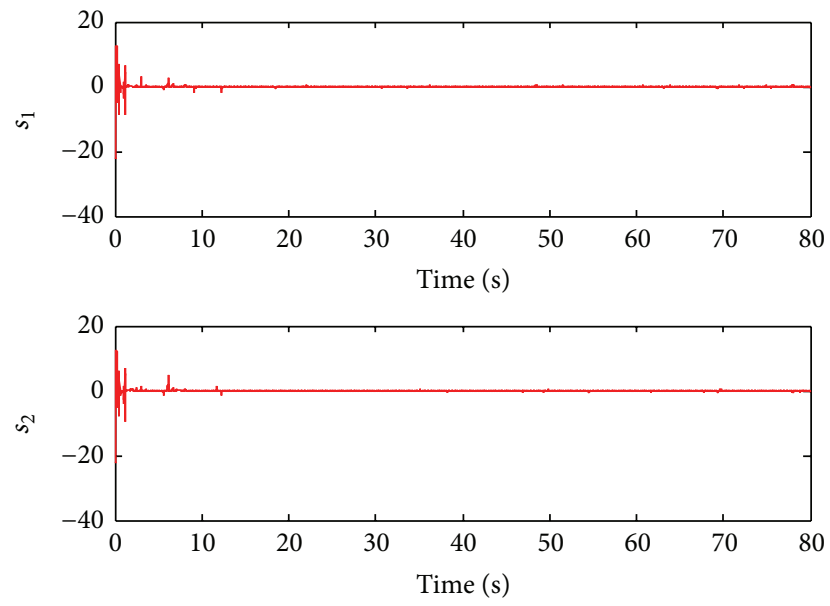

FIGURE 6: Switch functions using adaptive global sliding neural control.
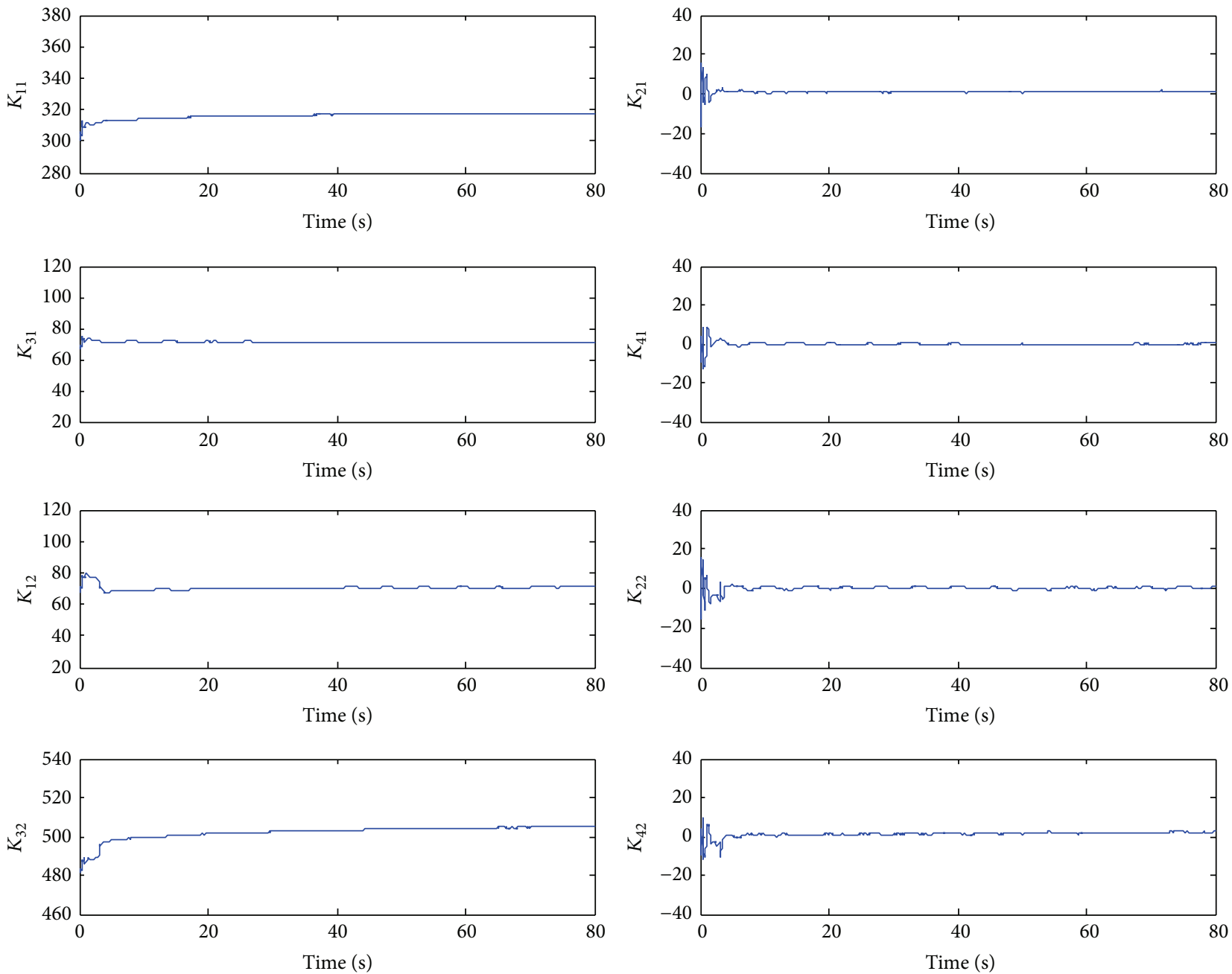

FIGURE 7: Adaptive estimates of unknown parameters using adaptive global sliding neural control. 


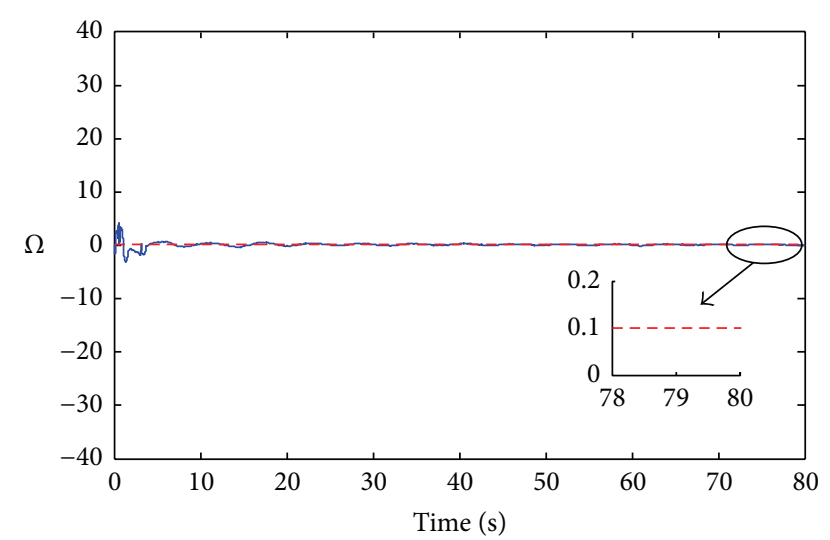

FIGURE 8: Estimate of angular velocity using adaptive global sliding neural control.
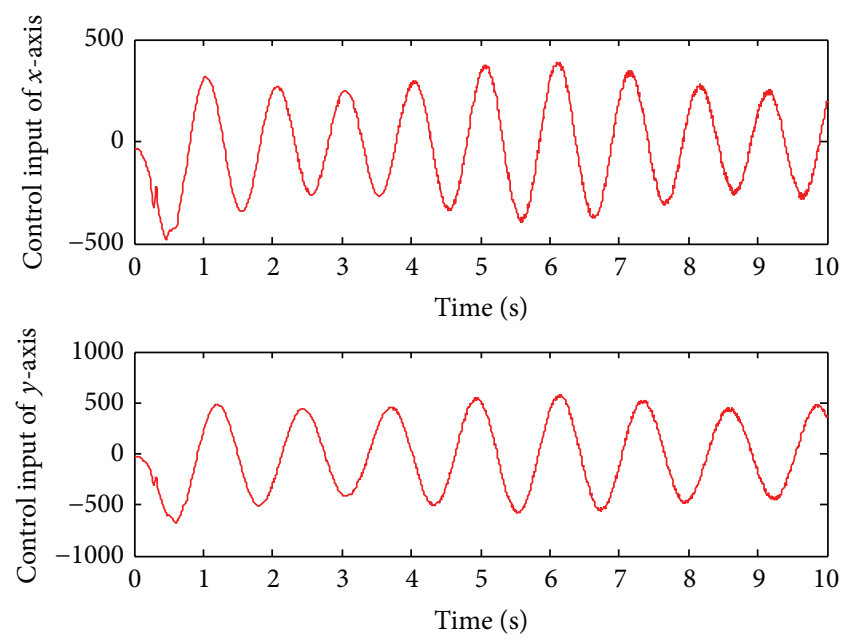

FIGURE 9: Control input adaptive global sliding neural control.

The dynamic global sliding mode surface is shown in Figure 6 which demonstrates that the sliding surfaces reach to zero asymptotically fast. It indicates that in such a short period of time the system gets to the switch surface and slides along the sliding surface, proving its effectiveness again. Figure 7 shows how the adaptive parameter $K$ changes with time $t$. It converges to its true value in a certain period of time to recognize the unknown parameters according to the simulation results. Figure 8 plots the angular velocity $\Omega_{z}$. We can also observe that it converges to its assumed value after the calculation of dimensionless value eventually. Therefore, it verifies that adaptive global sliding mode control is able to estimate the parameters quite accurately.

Figures 9 and 10 compare the control inputs between adaptive global sliding mode control using neural network and conventional fixed gain sliding control, respectively, where the estimate of the upper bound of lumped uncertainties and disturbances is obtained by adaptive global sliding mode control with neural network in Figure 9. The chattering in Figure 9 is obvious while it almost disappears in Figure 10. Chattering in adaptive global sliding mode
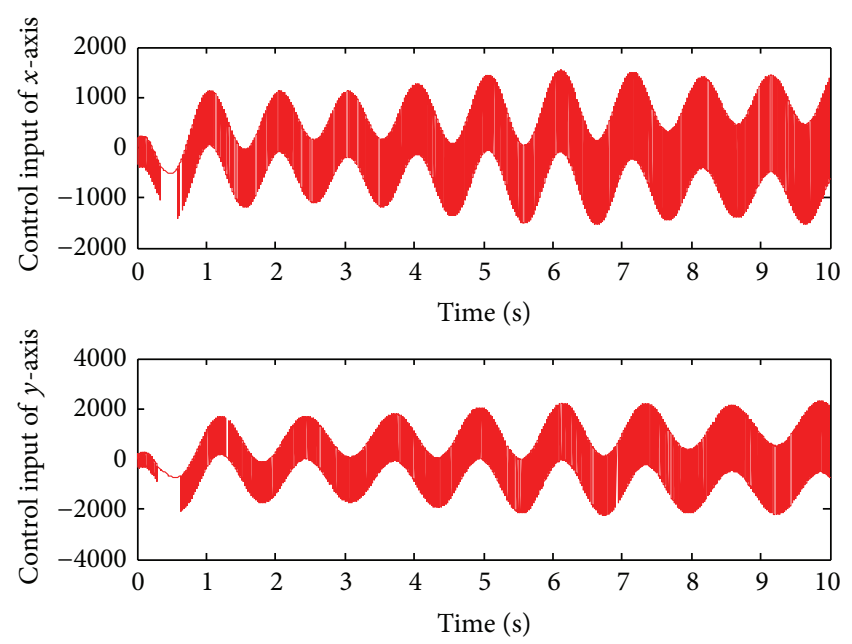

FIGURE 10: Control input under fixed sliding gain using global sliding mode control.

control using neural network is cut down largely since by the switch item in the sliding controller it is approached by the compensation function of neural network for the uncertainties and disturbances.

\section{Conclusion}

The global sliding mode control, adaptive control, and neural network control are combined to control the MEMS gyroscope. The adaptive global sliding mode algorithm is designed to guarantee the stability of the system and to make the real trajectory track the ideal trajectory rapidly. For unknown parameters in the system, we can take advantage of the adaptive estimator to identify the true value of the angular velocity and other parameters of MEMS gyroscope. The chattering can be alleviated greatly through the upper bound estimation of the lumped uncertainties and external disturbances by neural network. Simulation is implemented to verify that the proposed adaptive global sliding neural control method has satisfactory performance.

\section{Conflict of Interests}

The authors declare that there is no conflict of interests regarding the publication of this paper.

\section{Acknowledgments}

This work is partially supported by the National Science Foundation of China under Grant no. 61374100 and the Natural Science Foundation of Jiangsu Province under Grant no. BK20131136.

\section{References}

[1] C. Batur, T. Sreeramreddy, and Q. Khasawneh, "Sliding mode control of a simulated MEMS gyroscope," ISA Transactions, vol. 45, no. 1, pp. 99-108, 2006. 
[2] R. P. Leland, "Adaptive control of a MEMS gyroscope using lyapunov methods," IEEE Transactions on Control Systems Technology, vol. 14, no. 2, pp. 278-283, 2006.

[3] J. Fei, X. Fan, W. Dai, J. Shen, and M. Hua, "Robust tracking control of triaxial angular velocity sensors using adaptive sliding mode approach," International Journal of Advanced Manufacturing Technology, vol. 52, no. 5-8, pp. 627-636, 2011.

[4] S. Sung, W.-T. Sung, C. Kim, S. Yun, and Y. J. Lee, "On the mode-matched control of MEMS vibratory gyroscope via phase-domain analysis and design," IEEE/ASME Transactions on Mechatronics, vol. 14, no. 4, pp. 446-455, 2009.

[5] J. D. John and T. Vinay, "Novel concept of a single-mass adaptively controlled triaxial angular rate sensor," IEEE Sensors Journal, vol. 6, no. 3, pp. 588-595, 2006.

[6] L. Liu, Z. Han, and W. Li, "Global sliding mode control and application in chaotic systems," Nonlinear Dynamics, vol. 56, no. 1-2, pp. 193-198, 2009.

[7] D. Efimov and L. Fridman, "Global sliding-mode observer with adjusted gains for locally Lipschitz systems," Automatica, vol. 47, no. 3, pp. 565-570, 2011.

[8] Y. Chu and J. Fei, "Global sliding mode control of MEMS gyroscope based on neural network," in Proceedings of the 13th International Workshop on Advanced Motion Control (AMC '14), pp. 575-580, Yokohama, Japan, March 2014.

[9] D. Rastovic, "Compensators for contractive systems," Cybernetica, vol. 36, no. 1, pp. 11-16, 1993.

[10] X. Zhang, "Discrete adaptive sliding mode control via wavelet network for a class of nonlinear systems," Applied Mathematics \& Information Sciences, vol. 8, no. 6, pp. 3055-3062, 2014.

[11] C.-M. Lin and H.-Y. Li, "Adaptive dynamic sliding-mode fuzzy CMAC for voice coil motor using asymmetric gaussian membership function," IEEE Transactions on Industrial Electronics, vol. 61, no. 10, pp. 5662-5671, 2014.

[12] J. Fei and H. Ding, "Adaptive sliding mode control of dynamic system using RBF neural network," Nonlinear Dynamics, vol. 70, no. 2, pp. 1563-1573, 2012.

[13] D. Rastovic, "Targeting and synchronization at tokamak with recurrent artificial neural networks," Neural Computing and Applications, vol. 21, no. 5, pp. 1065-1069, 2012.

[14] J. Fei and J. Zhou, "Robust adaptive control of MEMS triaxial gyroscope using fuzzy compensator," IEEE Transactions on Systems, Man, and Cybernetics Part B: Cybernetics, vol. 42, no. 6, pp. 1599-1607, 2012.

[15] T.-C. Lin and M.-C. Chen, "Adaptive hybrid type-2 intelligent sliding mode control for uncertain nonlinear multivariable dynamical systems," Fuzzy Sets and Systems, vol. 171, no. 1, pp. $44-71,2011$.

[16] T. H. Oong and N. A. M. Isa, "Adaptive evolutionary artificial neural networks for pattern classification," IEEE Transactions on Neural Networks, vol. 22, no. 11, pp. 1823-1836, 2011.

[17] T. Zhang and S. S. Ge, "Adaptive neural network tracking control of MIMO nonlinear systems with unknown dead zones and control directions," IEEE Transactions on Neural Networks, vol. 20, no. 3, pp. 483-497, 2009.

[18] D. Zhao, W. Ni, and Q. Zhu, "A framework of neural networks based consensus control for multiple robotic manipulators," Neurocomputing, vol. 140, pp. 8-18, 2014.

[19] X. Lei and P. Lu, "The adaptive radial basis function neural network for small rotary-wing unmanned aircraft," IEEE Transactions on Industrial Electronics, vol. 61, no. 9, pp. 4808-4815, 2014.
[20] A.-M. Zou, K. D. Kumar, Z.-G. Hou, and X. Liu, "Finite-time attitude tracking control for spacecraft using terminal sliding mode and chebyshev neural network," IEEE Transactions on Systems, Man, and Cybernetics Part B: Cybernetics, vol. 41, no. 4, pp. 950-963, 2011.

[21] X.-Y. Lou and B.-T. Cui, "Robust adaptive synchronization of chaotic neural networks by slide technique," Chinese Physics B, vol. 17, no. 2, pp. 520-528, 2008.

[22] C.-S. Chen, "Dynamic structure neural-fuzzy networks for robust adaptive control of robot manipulators," IEEE Transactions on Industrial Electronics, vol. 55, no. 9, pp. 3402-3414, 2008. 


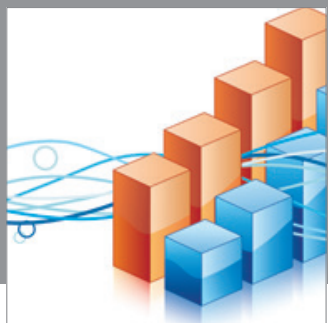

Advances in

Operations Research

mansans

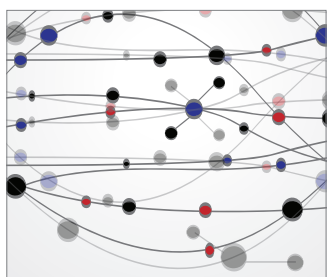

The Scientific World Journal
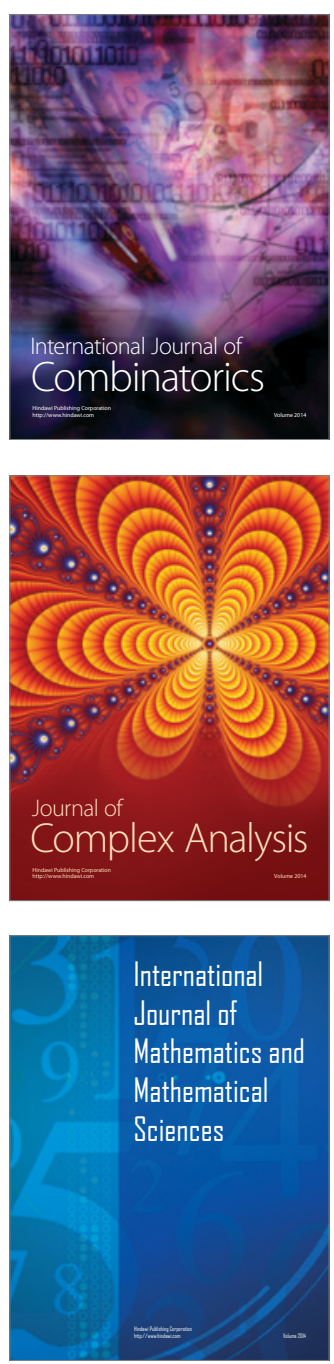
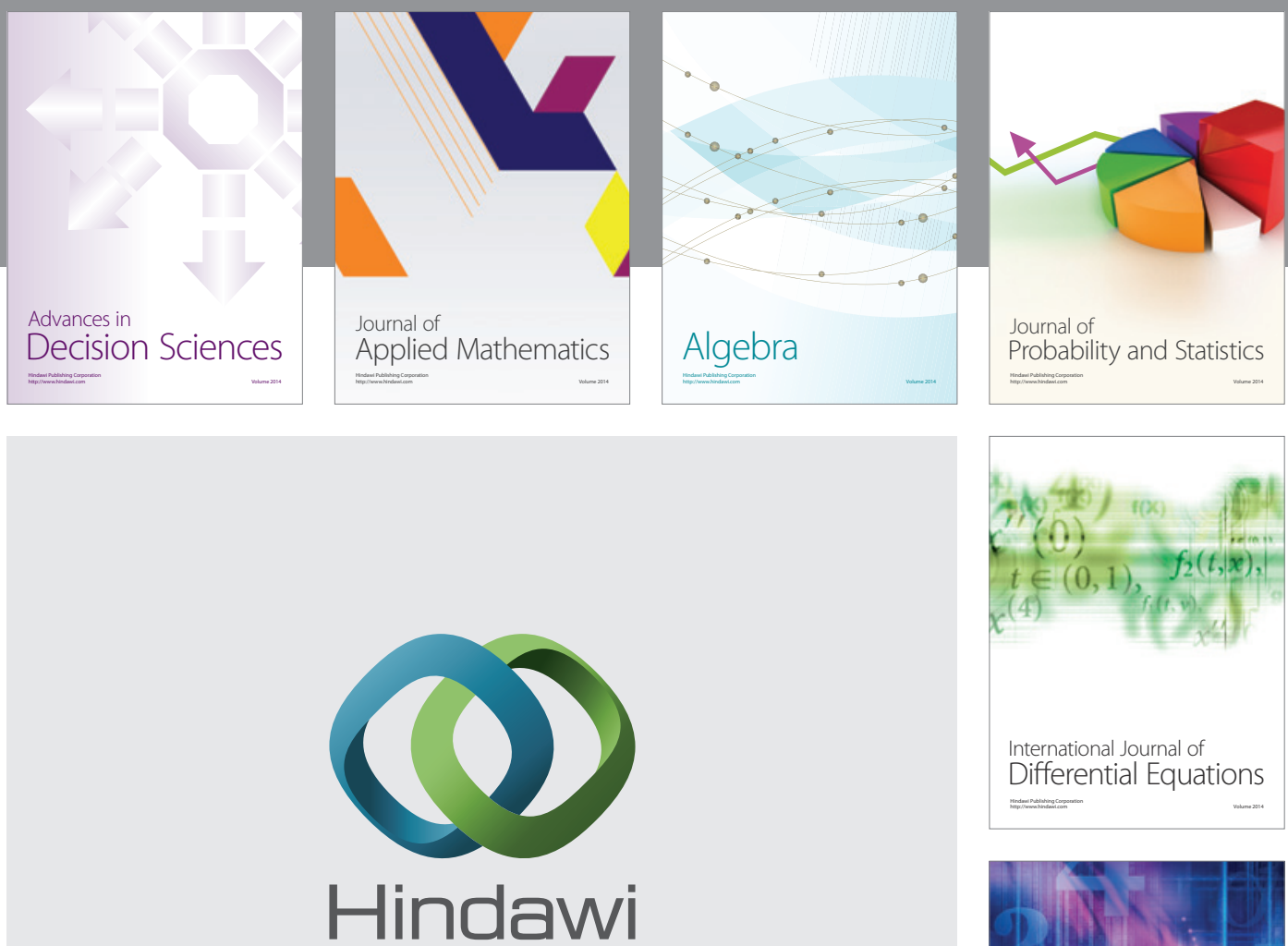

Submit your manuscripts at http://www.hindawi.com
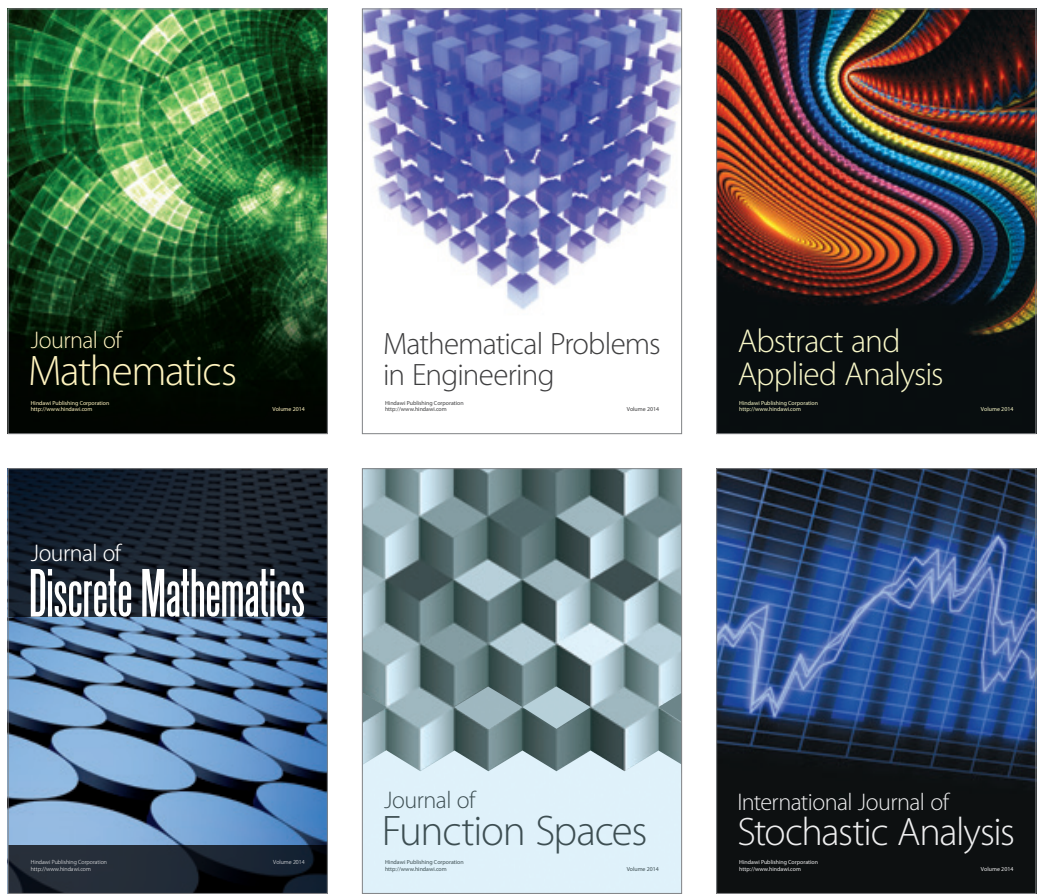

Journal of

Function Spaces

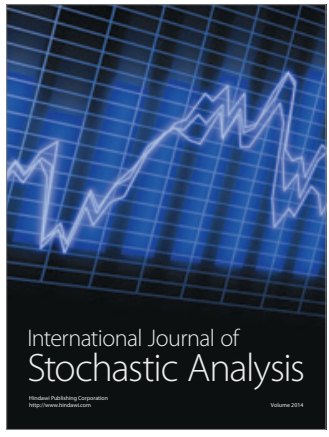

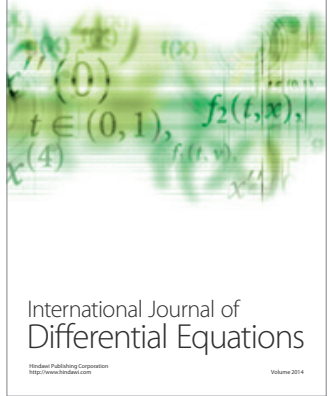
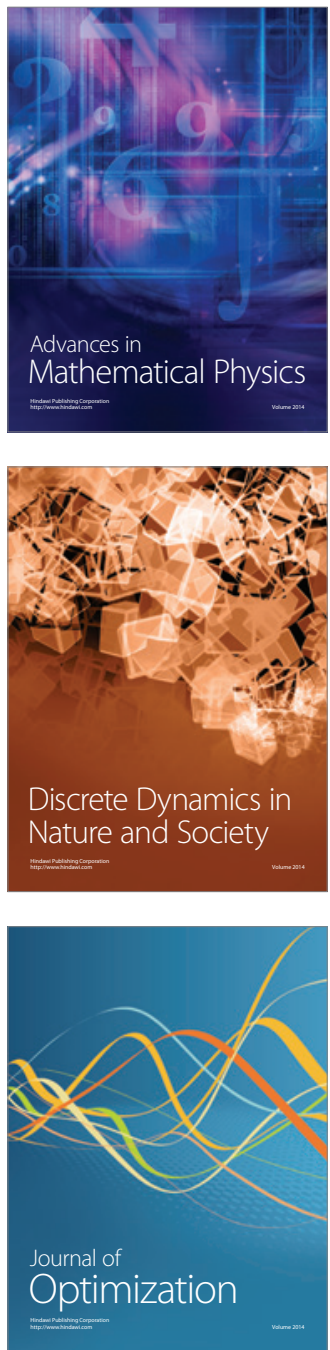